\title{
A synthetical two-component model with peakon solutions
}

\author{
Baoqiang Xia ${ }^{1,2 *}$, Zhijun Qiao ${ }^{2 \dagger}$ Ruguang Zhou ${ }^{1 \ddagger}$ \\ ${ }^{1}$ School of Mathematics and Statistics, Jiangsu Normal University, \\ Xuzhou, Jiangsu 221116, P. R. China \\ ${ }^{2}$ Department of Mathematics, University of Texas-Pan American, \\ Edinburg, Texas 78541, USA
}

\begin{abstract}
A generalized two-component model with peakon solutions is proposed in this paper. It allows an arbitrary function to be involved in as well as including some existing integrable peakon equations as special reductions. The generalized two-component system is shown to possess Lax pair and infinitely many conservation laws. Bi-Hamiltonian structures and peakon interactions are discussed in detail for typical representative equations of the generalized system. In particular, a new type of $N$-peakon solution, which is not in the traveling wave type, is obtained from the generalized system.
\end{abstract}

Keywords: Integrable system, Peakon, Lax pair.

MSC: $37 \mathrm{~K} 10,35 \mathrm{Q} 51$.

\footnotetext{
${ }^{*}$ E-mail address: xiabaoqiang@126.com

${ }^{\dagger}$ E-mail address: qiao@utpa.edu

${ }^{\ddagger}$ E-mail address: zhouruguang@jsnu.edu.cn
} 


\section{Introduction}

In recent years, the Camassa-Holm $(\mathrm{CH})$ equation [1]

$$
m_{t}=2 m u_{x}+m_{x} u, \quad m=u-u_{x x}+k,
$$

( $k$ is an arbitrary constant) derived by Camassa and Holm [1] as a shallow water wave model, has attracted much attention and various studies. The $\mathrm{CH}$ equation admits Lax representation [1], bi-Hamiltonian structure [2, 3], and is integrable by the inverse scattering transformation [7. Also it possesses multiple peaked soliton solutions [1, 4] and algebro-geometric solutions [5, 6]. The most interesting feature of the $\mathrm{CH}$ equation is that it admits peaked soliton (peakon) solutions in the case of $k=0$ [1, 4]. A peakon is a weak solution in some Sobolev space with corner at its crest. The stability and analysis study of peakons were discussed in several references [8]-12].

The interesting characteristics of the $\mathrm{CH}$ equation stimulated more people to search new integrable models which admit peakon solutions. Among them, for example, there are:

1. the Degasperis-Procesi (DP) equation [13]

$$
m_{t}=3 m u_{x}+m_{x} u, \quad m=u-u_{x x}
$$

which was shown integrable with Lax pair and bi-Hamiltonian structure in [14, multi-peakon dynamics in [15], and shocks formation in [16];

2. the cubic nonlinear peakon equation - the FORQ equation [17, 3, 18,

$$
m_{t}=\left[m\left(u^{2}-u_{x}^{2}\right)\right]_{x}, \quad m=u-u_{x x},
$$

which was shown to have Lax pair and bi-Hamiltonian structure in [19], and peakon solutions in [20];

3. the Novikov's cubic nonlinear equation 21

$$
m_{t}=u^{2} m_{x}+3 u u_{x} m, \quad m=u-u_{x x}
$$

which was shown integrable with Lax pair, bi-Hamiltonian structure and conservation laws in [22]; and

4. the generalized $\mathrm{CH}$ equation with both quadratic and cubic nonlinearity [17, 18, 23 ]

$$
m_{t}=\frac{1}{2} k_{1}\left[m\left(u^{2}-u_{x}^{2}\right)\right]_{x}+\frac{1}{2} k_{2}\left(2 m u_{x}+m_{x} u\right), \quad m=u-u_{x x},
$$

where $k_{1}$ and $k_{2}$ are two arbitrary constants. Equation (5) was proven to possess Lax pair, conservation laws and peakon solutions in [23]. 
Equation (5) is actually a linear combination of $\mathrm{CH}$ equation (11) and cubic nonlinear equation (3). This structure is very similar to the Gardner equation, known as a linear combination of $\mathrm{KdV}$ and $\mathrm{mKdV}$ equations. Thus equation (5) is the dual system of the Gardner equation from the viewpoint of tri-Hamiltonian duality [3, 18]. In the literature [17], a more generalized version of equation (5) was derived by Fokas from the two-dimensional hydrodynamical equations for surface waves. We also notice that by some appropriate rescaling or gauge transformations equation (5) is equivalent to equation (3).

All equations shown above are scalar integrable peakon models. Another important task is to find integrable multi-component peakon systems to enrich the theory of soliton and integrable systems. For example, the integrable two-component $\mathrm{CH}$ equations are proposed in [3, 24, 25, 26]. The integrable two-component forms of the cubic peakon systems (33) and (4) are presented in [27, 28, 29].

In addition to the integrable generalizations of peakon equations, there are also some works appearing to study the non-integrable generalizations of peakon equations. The most well-known example is the so-called $b$-family equation by Holm and Staley [30, 31]

$$
m_{t}=b m u_{x}+m_{x} u, \quad m=u-u_{x x},
$$

where $b$ is an arbitrary constant. The case of $b=2$ is exactly the $\mathrm{CH}$ equation, while the case of $b=3$ recovers the DP equation. According to various tests for integrability, it is known that the cases of $b=2$ and $b=3$ are the only integrable equations within this family [32]-35]. But for any $b$, all those equations admit peakon solutions [31]. Holm and Staley also studied peakon dynamics of (6) for different values of $b$ and discussed how they behave with changing $b$ [31]. In the literature [36], Popowicz proposed a two-component system, which can be considered as a coupling between the $\mathrm{CH}$ equation and the DP equation. Later, Hone and Irle showed that the two-component Popowicz system is non-integrable, but admits single-peakon solution as well as multi-peakon solutions [37.

In this paper, we propose the following generalized version of the two-component peakon system

$$
\left\{\begin{array}{l}
m_{t}=(m H)_{x}+m H-\frac{1}{2} m\left(u-u_{x}\right)\left(v+v_{x}\right), \\
n_{t}=(n H)_{x}-n H+\frac{1}{2} n\left(u-u_{x}\right)\left(v+v_{x}\right), \\
m=u-u_{x x}, \\
n=v-v_{x x},
\end{array}\right.
$$

where $H$ is an arbitrary function of $u, v$ and their derivatives. As $v=2$ and $H=u$, equation (7) is reduced to the $\mathrm{CH}$ equation (11). As $v=2 u$ and $H=\left(u^{2}-u_{x}^{2}\right)$, equation (7) is reduced to the FORQ equation (3). As $v=k_{1} u+k_{2}$ and $H=\frac{1}{2}\left[k_{1}\left(u^{2}-u_{x}^{2}\right)+k_{2} u\right]$, equation (7) is cast into the generalized $\mathrm{CH}$ equation (5). Thus, equation (17) is a kind of the two-component 
generalization of equations (11), (3) and (5). We show that the generalized system (7) possesses an $\operatorname{sl}(2)$-valued Lax pair and infinitely many conservation laws. Since the arbitrary function $H$ is involved in (7), we do not expect all those equations have bi-Hamiltonian structures in general. Nevertheless, we demonstrate that for some special choices of $H$ we may find the corresponding bi-Hamiltonian structures. Such a system is interesting, because we may obtain quite a large number of integrable peakon equations by choosing different $H$. We take some examples to discuss in detail the bi-Hamiltonian structures and the peakon interactions for some equations in the family (7). From the equations in the family (7), we obtain a new type of $N$-peakon solution which is not presented in the traveling wave type.

The whole paper is organized as follows. Section 2 provides the Lax pair and conservation laws for the system (7). Section 3 studies the bi-Hamiltonian structures and the multi-peakon solutions of some two-component equations in the family (77). Section 4 supplies a proof for the bi-Hamiltonian property in each example discussed in section 3. Some conclusions and open problems are addressed in section 5 .

\section{Lax pair and conservation laws}

Let us consider a pair of $2 \times 2$ matrix spectral problems of the following type

$$
\begin{aligned}
& \phi_{x}=U \phi, \quad U=\frac{1}{2}\left(\begin{array}{cc}
-1 & \lambda m \\
-\lambda n & 1
\end{array}\right), \\
& \phi_{t}=V \phi, \quad V=-\frac{1}{2}\left(\begin{array}{cc}
\lambda^{-2}+\frac{1}{2}\left(u-u_{x}\right)\left(v+v_{x}\right) & -\lambda^{-1}\left(u-u_{x}\right)-\lambda m H \\
\lambda^{-1}\left(v+v_{x}\right)+\lambda n H & -\lambda^{-2}-\frac{1}{2}\left(u-u_{x}\right)\left(v+v_{x}\right)
\end{array}\right),
\end{aligned}
$$

where

$$
\phi=\left(\phi_{1}, \phi_{2}\right)^{T}, \quad m=u-u_{x x}, \quad n=v-v_{x x},
$$

$\lambda$ is a spectral parameter and $H$ is an arbitrary function of $u, v$ and their derivatives.

It is easy to see that the compatibility condition of (8) and (9) reads

$$
U_{t}-V_{x}+[U, V]=0 .
$$

Substituting the expressions of (8) and (9) into (11), we immediately find that (11) is nothing but equation (7). Thus, (8) and (9) compose of a Lax pair of equation (7).

Remark 1. Our generalized system with an arbitrary function $H$ involved does admit an $s l(2)$-valued Lax representation. System (7) is produced by the compatibility condition (11) of the spectral problems (8) and (9) where such an arbitrary function is included in $V$ part. 
The arbitrary function $H$ is able to appear because the Lax equation (11) is an overdetermined system by choosing the appropriate $V$ (dependent on $\lambda$ ) to match $U$.

Next, let us construct the conservation laws for system (7) by using spectral problems (8) and (9). Let $\omega=\frac{\phi_{2}}{\phi_{1}}$, then it follows from (8) that $\omega$ satisfies the Riccati equation

$$
\omega_{x}=-\frac{1}{2} \lambda m \omega^{2}+\omega-\frac{1}{2} \lambda n .
$$

Based on (8) and (9), we obtain

$$
\begin{aligned}
\left(\ln \phi_{1}\right)_{x} & =-\frac{1}{2}+\frac{1}{2} \lambda m \omega, \\
\left(\ln \phi_{1}\right)_{t} & =-\frac{1}{2}\left[\lambda^{-2}-\lambda^{-1}\left(u-u_{x}\right) \omega+\frac{1}{2}\left(u-u_{x}\right)\left(v+v_{x}\right)-\lambda m H \omega\right],
\end{aligned}
$$

which generates the following conservation law of equation (7)

$$
\rho_{t}=A_{x}
$$

where

$$
\begin{aligned}
\rho & =m \omega \\
A & =-\frac{1}{2} \lambda^{-1}\left(u-u_{x}\right)\left(v+v_{x}\right)+\lambda^{-2}\left(u-u_{x}\right) \omega+m H \omega .
\end{aligned}
$$

Usually $\rho$ and $A$ are called a conserved density and an associated flux, respectively.

We are able to derive the explicit forms of conservation densities by expanding $\omega$ in powers of $\lambda$ in two ways. The first one is to expand $\omega$ in terms of negative powers of $\lambda$ as

$$
\omega=\sum_{j=0}^{\infty} \omega_{j} \lambda^{-j} .
$$

By substituting (16) into (12) and equating the coefficients of powers of $\lambda$, we arrive at

$$
\begin{aligned}
\omega_{0} & =\sqrt{-\frac{n}{m}}, \quad \omega_{1}=\frac{m n_{x}-m_{x} n-2 m n}{2 m^{2} n}, \\
\omega_{j+1} & =\frac{1}{m \omega_{0}}\left[\omega_{j}-\omega_{j, x}-\frac{1}{2} m \sum_{i+k=j+1,1 \leq i, k \leq j} \omega_{i} \omega_{k}\right], \quad j \geq 1 .
\end{aligned}
$$

Inserting (16) and (17) into (15), we obtain the following infinitely many conserved densities and the associated fluxes

$$
\begin{aligned}
& \rho_{0}=\sqrt{-m n}, \quad A_{0}=H \sqrt{-m n}, \\
& \rho_{1}=\frac{m n_{x}-m_{x} n-2 m n}{2 m n}, \quad A_{1}=-\frac{1}{2}\left(u-u_{x}\right)\left(v+v_{x}\right)+\frac{\left(m n_{x}-m_{x} n-2 m n\right) H}{2 m n}, \\
& \rho_{j}=m \omega_{j}, \quad A_{j}=\left(u-u_{x}\right) \omega_{j-2}+m H \omega_{j}, \quad j \geq 2
\end{aligned}
$$


where $\omega_{j}$ is given by (17).

The second expansion of $\omega$ is in the positive powers of $\lambda$ as

$$
\omega=\sum_{j=0}^{\infty} \omega_{j} \lambda^{j}
$$

Substituting (19) into (12) and comparing powers of $\lambda$ lead to

$$
\begin{aligned}
\omega_{2 j} & =0, \quad j \geq 0, \\
\omega_{1} & =\frac{1}{2}\left(v+v_{x}\right), \quad \omega_{2 j+1}-\omega_{2 j+1, x}=\frac{1}{2} m \sum_{i+k=2 j, 0 \leq i, k \leq 2 j} \omega_{i} \omega_{k}, \quad j \geq 1 .
\end{aligned}
$$

From formula (201), we know

$$
\rho_{2 j}=0, \quad A_{2 j}=0, \quad j \geq 0,
$$

which means the even-index conserved densities and associated fluxes are trivial. From formula (21), we arrive at the odd-index conserved densities and associated fluxes

$$
\begin{aligned}
\rho_{1} & =\frac{1}{2} m\left(v+v_{x}\right), \quad A_{1}=\left(u-u_{x}\right) \omega_{3}+\frac{1}{2} m\left(v+v_{x}\right) H, \\
\rho_{2 j+1} & =m \omega_{2 j+1}, \quad A_{2 j+1}=\left(u-u_{x}\right) \omega_{2 j+3}+m H \omega_{2 j+1}, \quad j \geq 1,
\end{aligned}
$$

where the odd-index $\omega_{2 j+1}$ is defined by the recursion relation

$$
\omega_{2 j+1}=\frac{1}{2}\left(1-\partial_{x}\right)^{-1}\left(m \sum_{i+k=2 j, 0 \leq i, k \leq 2 j} \omega_{i} \omega_{k}\right), \quad j \geq 1 .
$$

We should remark that the relation (24) shows the nontrivial high-order conserved densities in the sequence (23) may involve in nonlocal expressions in $u$ and $v$. However, the conserved densities in the sequence (18) are local ones.

Remark 2. The expressions (18) and (23) show that all members in our generalized system possess the same conserved quantities but different conserved fluxes. This is because the conserved quantities are derived from the Riccati equation (12) that only depends on the spatial part of the Lax representation which keeps the same for all members in the family; while the conserved fluxes rely on the temporal part of the Lax representation which changes for different members.

\section{Two-component peakon systems}

The two-component system (7) is of great interest because different choices of $H$ lead to different peakon equations. Let us discuss some special cases in the following examples. 


\section{Example 1. A new integrable system with a new type of peakon solutions}

Taking $H=0$ in equation (7) gives rise to the following integrable two-component model

$$
\left\{\begin{array}{l}
m_{t}=-\frac{1}{2} m\left(u-u_{x}\right)\left(v+v_{x}\right) \\
n_{t}=\frac{1}{2} n\left(u-u_{x}\right)\left(v+v_{x}\right) \\
m=u-u_{x x}, \quad n=v-v_{x x}
\end{array}\right.
$$

This model can be rewritten as the following bi-Hamiltonian form

$$
\left(m_{t}, n_{t}\right)^{T}=J\left(\frac{\delta H_{2}}{\delta m}, \frac{\delta H_{2}}{\delta n}\right)^{T}=K\left(\frac{\delta H_{1}}{\delta m}, \frac{\delta H_{1}}{\delta n}\right)^{T}
$$

where

$$
\begin{aligned}
J & =\left(\begin{array}{cc}
0 & -\partial-1 \\
-\partial+1 & 0
\end{array}\right), K=\left(\begin{array}{cc}
-m \partial^{-1} m & m \partial^{-1} n \\
n \partial^{-1} m & -n \partial^{-1} n
\end{array}\right), \\
H_{1} & =\frac{1}{2} \int_{-\infty}^{+\infty} m\left(v+v_{x}\right) d x, \quad H_{2}=\frac{1}{4} \int_{-\infty}^{+\infty}\left(u-u_{x}\right)^{2}\left(v+v_{x}\right) n d x
\end{aligned}
$$

In section 4 , we will provide a detailed proof for the compatibility of the Hamiltonian pairs $J$ and $K$ in this example and the next three examples.

Let us assume that (25) has the following one-peakon solution

$$
u=p_{1}(t) e^{-\left|x-q_{1}(t)\right|}, \quad v=r_{1}(t) e^{-\left|x-q_{1}(t)\right|},
$$

where $p_{1}(t), r_{1}(t)$ and $q_{1}(t)$ are functions of $t$ needed to be determined. Substituting (29) into (25) and integrating against the test function with support around the peak, we obtain

$$
p_{1, t}=-\frac{1}{3} p_{1}^{2} r_{1}, \quad r_{1, t}=\frac{1}{3} p_{1} r_{1}^{2}, \quad q_{1, t}=0
$$

which yields

$$
p_{1}(t)=A_{2} e^{-\frac{1}{3} A_{1} t}, \quad r_{1}(t)=\frac{A_{1}}{A_{2}} e^{\frac{1}{3} A_{1} t}, \quad q_{1}(t)=A_{3},
$$

where $A_{1}, A_{2}$, and $A_{3}$ are integration constants. Thus, we obtain the peakon solutions as follows

$$
u(x, t)=A_{2} e^{-\frac{1}{3} A_{1} t} e^{-\left|x-A_{3}\right|}, \quad v(x, t)=\frac{A_{1}}{A_{2}} e^{\frac{1}{3} A_{1} t} e^{-\left|x-A_{3}\right|} .
$$

This pair of single-peakon solutions is not presented in the traveling wave type, because the peakon position $q_{1}(t)=A_{3}$ is stationary. To the best of our knowledge, almost all integrable peakon models have single peakons which are of traveling wave type. So, we find a new integrable peakon system (25) whose peakon solution is not in traveling wave type. See Figure 1 for the profile of the new single-peakon solution. We remark that the amplitudes of the peakons 
of equation (25) grow/decay exponentially with time. Recently, Lundmark and Szmigielski [38] found that the Geng-Xue two-component system [29] has a similar type of peakons (with amplitudes exponentially growing/decaying with time).

Let us suppose the $N$-peakon solution in the form of

$$
u(x, t)=\sum_{j=1}^{N} p_{j}(t) e^{-\left|x-q_{j}(t)\right|}, \quad v(x, t)=\sum_{j=1}^{N} r_{j}(t) e^{-\left|x-q_{j}(t)\right|} .
$$

By substituting (33) into (25) and integrating against test functions, we obtain the $N$-peakon dynamic system of (25)

$$
\left\{\begin{array}{l}
q_{j, t}=0 \\
p_{j, t}=\frac{1}{6} p_{j}^{2} r_{j}+\frac{1}{2} p_{j} \sum_{i, k=1}^{N} p_{i} r_{k}\left(\operatorname{sgn}\left(q_{j}-q_{i}\right)+1\right)\left(\operatorname{sgn}\left(q_{j}-q_{k}\right)-1\right) e^{-\left|q_{j}-q_{i}\right|-\left|q_{j}-q_{k}\right|} \\
r_{j, t}=-\frac{1}{6} p_{j} r_{j}^{2}-\frac{1}{2} r_{j} \sum_{i, k=1}^{N} p_{i} r_{k}\left(\operatorname{sgn}\left(q_{j}-q_{i}\right)+1\right)\left(\operatorname{sgn}\left(q_{j}-q_{k}\right)-1\right) e^{-\left|q_{j}-q_{i}\right|-\left|q_{j}-q_{k}\right|} .
\end{array}\right.
$$

In the above formula, $q_{j, t}=0$ implies that the peak position does not change along with the time $t$.

For $N=2$, solving (34) leads to

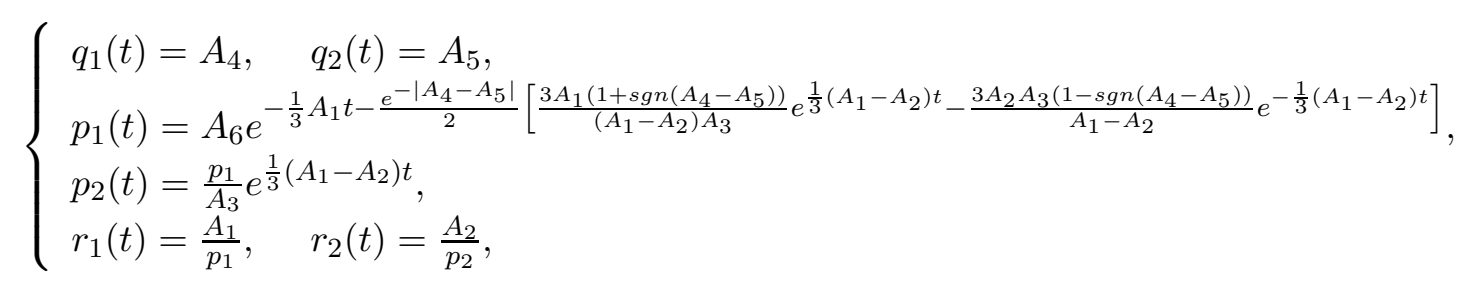

where $A_{1}, A_{2}, \cdots, A_{6}$ are integration constants. If $A_{4}=A_{5}$, it is reduced to the one-peakon solution. If $A_{4} \neq A_{5}$, this two-peakon solution will never collide because $q_{1}(t) \neq q_{2}(t)$ for any $t$. In particular, for $A_{1}=A_{3}=A_{4}=-A_{5}=A_{6}=1$ and $A_{2}=4$, the two-peakon becomes

$$
\left\{\begin{array}{l}
u(x, t)=e^{-\frac{1}{3} t+e^{-t-2}} e^{-|x-1|}+e^{-\frac{4}{3} t+e^{-t-2}} e^{-|x+1|} \\
v(x, t)=e^{\frac{1}{3} t-e^{-t-2}} e^{-|x-1|}+4 e^{\frac{4}{3} t-e^{-t-2}} e^{-|x+1|}
\end{array}\right.
$$

See Figure 2 for the profile of the two-peakon dynamics for the potentials $u(x, t)$ and $v(x, t)$.

Remark 3. We point out that from (25) one may conclude $(m n)_{t}=0$ and thus $m n=f(x)$, where $f(x)$ is a free function of $x$. It then follows that $v=\left(1-\partial^{2}\right)^{-1}\left(\frac{f(x)}{m}\right)$. This means we can remove the component $v$ in equation (25) and thus write (25) in the form of a single field equation. However, the resulting single field equation involves in nonlocal expressions and a free 


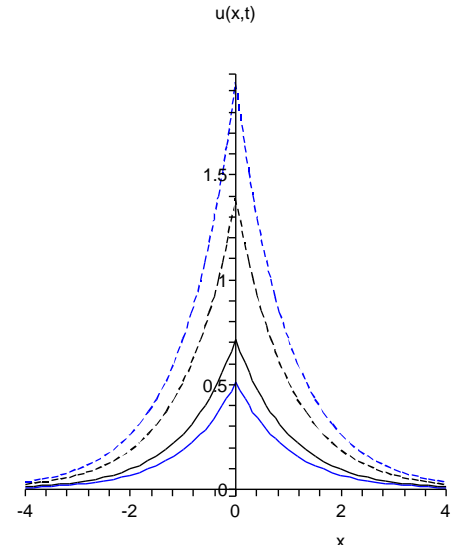

Figure 1: The single-peakon solution given by (32) with $A_{1}=A_{2}=1$ and $A_{3}=0$. Solid line: $u(x, t)$; Dashed line: $v(x, t)$; Black: $t=1$; Blue: $t=2$.

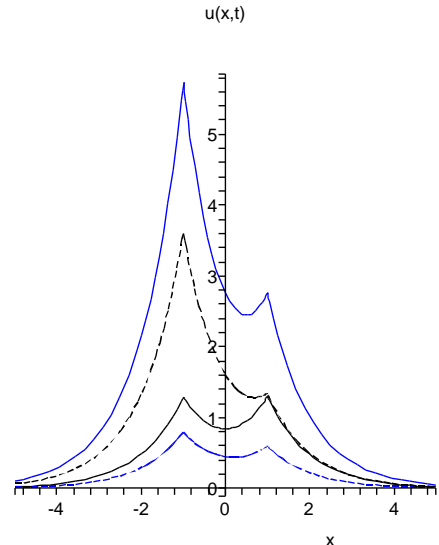

Figure 2: The two-peakon solution given by (36). Solid line: $u(x, t)$; Dashed line: $v(x, t)$; Black: $t=0$; Blue: $t=-1$.

function $f(x)$. Guided by this, we still write the equation associated with the case of $H=0$ in the form of (25).

Example 2. The integrable two-component system proposed in [28]

By choosing $H=\frac{1}{2}\left(u v-u_{x} v_{x}\right)$, we obtain

$$
\left\{\begin{array}{l}
m_{t}=\frac{1}{2}\left[m\left(u v-u_{x} v_{x}\right)\right]_{x}-\frac{1}{2} m\left(u v_{x}-u_{x} v\right) \\
n_{t}=\frac{1}{2}\left[n\left(u v-u_{x} v_{x}\right)\right]_{x}+\frac{1}{2} n\left(u v_{x}-u_{x} v\right) \\
m=u-u_{x x}, \quad n=v-v_{x x}
\end{array}\right.
$$

which is exactly the dispersionless version of the system we derived in [28]. This system possesses the bi-Hamiltonian form

$$
\left(m_{t}, n_{t}\right)^{T}=J\left(\frac{\delta H_{2}}{\delta m}, \frac{\delta H_{2}}{\delta n}\right)^{T}=K\left(\frac{\delta H_{1}}{\delta m}, \frac{\delta H_{1}}{\delta n}\right)^{T},
$$

where

$$
\begin{aligned}
J & =\left(\begin{array}{cc}
0 & \partial^{2}-1 \\
1-\partial^{2} & 0
\end{array}\right), K=\left(\begin{array}{cc}
\partial m \partial^{-1} m \partial-m \partial^{-1} m & \partial m \partial^{-1} n \partial+m \partial^{-1} n \\
\partial n \partial^{-1} m \partial+n \partial^{-1} m & \partial n \partial^{-1} n \partial-n \partial^{-1} n
\end{array}\right), \\
H_{1} & =\frac{1}{2} \int_{-\infty}^{+\infty}\left(u v+u_{x} v_{x}\right) d x, \quad H_{2}=\frac{1}{4} \int_{-\infty}^{+\infty}\left(u^{2} v_{x}+u_{x}^{2} v_{x}-2 u u_{x} v\right) n d x .
\end{aligned}
$$

In [28], we have derived the one-peakon of (37)

$$
u(x, t)=c_{1} e^{-\left|x+\frac{1}{3} c_{1} c_{2} t\right|}, \quad v(x, t)=c_{2} e^{-\left|x+\frac{1}{3} c_{1} c_{2} t\right|},
$$


where $c_{1}$ and $c_{2}$ are two arbitrary integration constants. We also investigated the $N$-peakon dynamical system. In particular, the two-peakon solution was given explicitly and the collisions are discussed (for details, see [28]).

Example 3. A new integrable two-component peakon system with the same bi-Hamiltonian operators as (37) but different Hamiltonian functions

Taking $H=\frac{1}{2}\left(u v_{x}-u_{x} v\right)$, we arrive at

$$
\left\{\begin{array}{l}
m_{t}=\frac{1}{2}\left[m\left(u v_{x}-u_{x} v\right)\right]_{x}-\frac{1}{2} m\left(u v-u_{x} v_{x}\right) \\
n_{t}=\frac{1}{2}\left[n\left(u v_{x}-u_{x} v\right)\right]_{x}+\frac{1}{2} n\left(u v-u_{x} v_{x}\right) \\
m=u-u_{x x}, \quad n=v-v_{x x}
\end{array}\right.
$$

This system can be rewritten as the following bi-Hamiltonian form

$$
\left(m_{t}, n_{t}\right)^{T}=J\left(\frac{\delta H_{2}}{\delta m}, \frac{\delta H_{2}}{\delta n}\right)^{T}=K\left(\frac{\delta H_{1}}{\delta m}, \frac{\delta H_{1}}{\delta n}\right)^{T}
$$

where $J, K$ are given by (39), and

$$
H_{1}=\frac{1}{2} \int_{-\infty}^{+\infty}\left(u v_{x}+u_{x} v_{x x}\right) d x, \quad H_{2}=\frac{1}{4} \int_{-\infty}^{+\infty}\left(u^{2} v+u_{x}^{2} v-2 u u_{x} v_{x}\right) n d x
$$

From (38) and (43), we find that equation (37) and (42) share the same bi-Hamiltonian operators but with different Hamiltonian functions. We will comment this at the end of this example (see Remark 4 below).

Let us study the peakon solutions of this example. By direct calculations, we find that the one-peakon solution of (42) takes the form as

$$
u(x, t)=c_{2} e^{-\frac{1}{3} c_{1} t} e^{-\left|x-c_{3}\right|}, \quad v(x, t)=\frac{c_{1}}{c_{2}} e^{\frac{1}{3} c_{1} t} e^{-\left|x-c_{3}\right|}
$$

where $c_{1}, c_{2}$ and $c_{3}$ are three integration constants. In general, we suppose the $N$-peakon solution of (42) in the form of (33). Then we obtain the $N$-peakon dynamical system of (42)

$$
\left\{\begin{array}{l}
p_{j, t}=\frac{1}{6} p_{j}^{2} r_{j}+\frac{1}{2} p_{j} \sum_{i, k=1}^{N} p_{i} r_{k}\left(\operatorname{sgn}\left(q_{j}-q_{i}\right) \operatorname{sgn}\left(q_{j}-q_{k}\right)-1\right) e^{-\left|q_{j}-q_{i}\right|-\left|q_{j}-q_{k}\right|} \\
r_{j, t}=-\frac{1}{6} p_{j} r_{j}^{2}-\frac{1}{2} r_{j} \sum_{i, k=1}^{N} p_{i} r_{k}\left(\operatorname{sgn}\left(q_{j}-q_{i}\right) \operatorname{sgn}\left(q_{j}-q_{k}\right)-1\right) e^{-\left|q_{j}-q_{i}\right|-\left|q_{j}-q_{k}\right|} \\
q_{j, t}=\frac{1}{2} \sum_{i, k=1}^{N} p_{i} r_{k}\left(\operatorname{sgn}\left(q_{j}-q_{k}\right)-\operatorname{sgn}\left(q_{j}-q_{i}\right)\right) e^{-\left|q_{j}-q_{i}\right|-\left|q_{j}-q_{k}\right|}
\end{array}\right.
$$


For $N=2$, the two-peakon dynamical system reads as

$$
\left\{\begin{array}{l}
p_{1, t}=-\frac{1}{3} p_{1}^{2} r_{1}-\frac{1}{2} p_{1}\left(p_{1} r_{2}+p_{2} r_{1}\right) e^{-\left|q_{1}-q_{2}\right|} \\
p_{2, t}=-\frac{1}{3} p_{2}^{2} r_{2}-\frac{1}{2} p_{2}\left(p_{1} r_{2}+p_{2} r_{1}\right) e^{-\left|q_{1}-q_{2}\right|} \\
r_{1, t}=\frac{1}{3} p_{1} r_{1}^{2}+\frac{1}{2} r_{1}\left(p_{1} r_{2}+p_{2} r_{1}\right) e^{-\left|q_{1}-q_{2}\right|} \\
r_{2, t}=\frac{1}{3} p_{2} r_{2}^{2}+\frac{1}{2} r_{2}\left(p_{1} r_{2}+p_{2} r_{1}\right) e^{-\left|q_{1}-q_{2}\right|} \\
q_{1, t}=\frac{1}{2}\left(p_{1} r_{2}-p_{2} r_{1}\right) \operatorname{sgn}\left(q_{1}-q_{2}\right) e^{-\left|q_{1}-q_{2}\right|} \\
q_{2, t}=q_{1, t}
\end{array}\right.
$$

From the first four equations of (47), we may conclude $p_{1}(t) r_{1}(t)=A_{1}$ and $p_{2}(t) r_{2}(t)=A_{2}$ where $A_{1}$ and $A_{2}$ are two integration constants. From the last two equations of (47), we know $q_{2}(t)=q_{1}(t)-B_{1}$ where $B_{1}$ is a nonzero constant, which indicates that the two-peakon will never collide. For $A_{1}=A_{2}$, we have

$$
\left\{\begin{array}{l}
p_{1}(t)=D e^{\left[-\frac{1}{3} A_{1}-\frac{1}{2}\left(A_{1} C_{1}+\frac{A_{1}}{C_{1}}\right) e^{-\left|B_{1}\right|}\right] t} \\
p_{2}(t)=\frac{p_{1}(t)}{C_{1}}, \quad r_{1}(t)=\frac{A_{1}}{p_{1}(t)}, \quad r_{2}=\frac{A_{1} C_{1}}{p_{1}(t)} \\
q_{1}(t)=\frac{1}{2}\left[\left(A_{1} C_{1}-\frac{A_{1}}{C_{1}}\right) \operatorname{sgn}\left(B_{1}\right) e^{\left.-\left|B_{1}\right|\right]} t+\frac{B_{1}}{2}\right. \\
q_{2}(t)=q_{1}(t)-B_{1},
\end{array}\right.
$$

where $B_{1}, C_{1}$, and $D$ are three integration constants. For example, choosing $C_{1}=D=1$, $B_{1}=2, A_{1}=3$, we have $p_{2}(t)=p_{1}(t)=e^{-\left(3 e^{-2}+1\right) t}, \quad r_{2}(t)=r_{1}(t)=3 e^{\left(3 e^{-2}+1\right) t}$. Thus, the two-peakon solution accordingly reads as

$$
\left\{\begin{array}{l}
u(x, t)=e^{-\left(3 e^{-2}+1\right) t}\left(e^{-|x-1|}+e^{-|x+1|}\right) \\
v(x, t)=3 e^{\left(3 e^{-2}+1\right) t}\left(e^{-|x-1|}+e^{-|x+1|}\right)
\end{array}\right.
$$

which are apparently M-shape peakon solutions with two peaks (see Figure 3 for details). If choosing $C_{1}=B_{1}=2, D=1, A_{1}=3$, then we have the following two-peakon solution

$$
\left\{\begin{array}{l}
u(x, t)=\frac{1}{2} e^{-\left(\frac{15}{4} e^{-2}+1\right) t}\left(2 e^{-\left|x-\frac{9}{4} e^{-2} t-1\right|}+e^{-\left|x-\frac{9}{4} e^{-2} t+1\right|}\right), \\
v(x, t)=3 e^{\left(\frac{15}{4} e^{-2}+1\right) t}\left(e^{-\left|x-\frac{9}{4} e^{-2} t-1\right|}+2 e^{-\left|x-\frac{9}{4} e^{-2} t+1\right|}\right) .
\end{array}\right.
$$

Figure 4 shows the profile of this two-peakon solution.

For $A_{1} \neq A_{2}$, we obtain the following solution of (47)

$$
\left\{\begin{array}{l}
p_{1}(t)=B_{3} e^{-\frac{1}{3} A_{1} t-\frac{3 e^{-\left|B_{1}\right|}}{2\left(A_{1}-A_{2}\right)}\left(\frac{A_{1}}{B_{2}} e^{\frac{1}{3}\left(A_{1}-A_{2}\right) t}-A_{2} B_{2} e^{-\frac{1}{3}\left(A_{1}-A_{2}\right) t}\right)} \\
p_{2}(t)=\frac{p_{1}}{B_{2}} e^{\frac{1}{3}\left(A_{1}-A_{2}\right) t} \\
r_{1}(t)=\frac{A_{1}}{p_{1}}, \quad r_{2}=\frac{A_{2}}{p_{2}} \\
q_{1}(t)=-\frac{3 \operatorname{sgn}\left(B_{1}\right) e^{\left|B_{1}\right|}}{2\left(A_{1}-A_{2}\right)}\left[A_{2} B_{2} e^{-\frac{1}{3}\left(A_{1}-A_{2}\right) t}+\frac{A_{1}}{B_{2}} e^{\frac{1}{3}\left(A_{1}-A_{2}\right) t}\right]+B_{4}, \\
q_{2}(t)=q_{1}-B_{1},
\end{array}\right.
$$




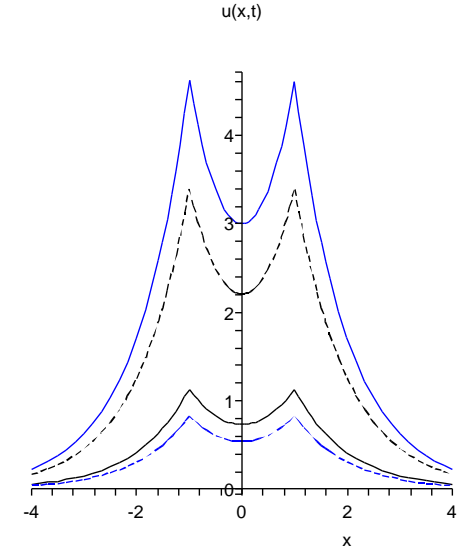

Figure 3: The M-shape peakon solution given by (49). Solid line: $u(x, t)$; Dashed line: $v(x, t) ;$ Black: $t=0$; Blue: $t=-1$.

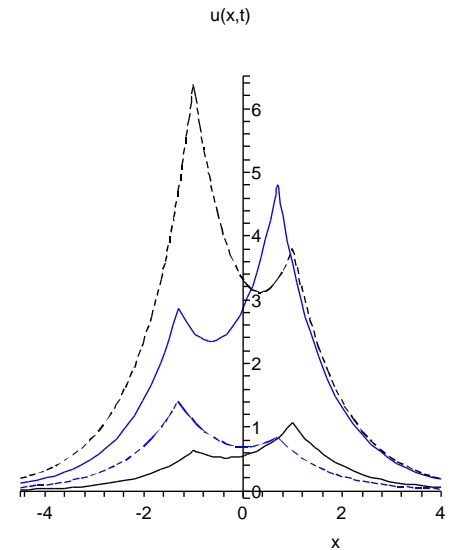

Figure 4: The two-peakon solution given by (50). Solid line: $u(x, t)$; Dashed line: $v(x, t)$; Black: $t=0$; Blue: $t=-1$.

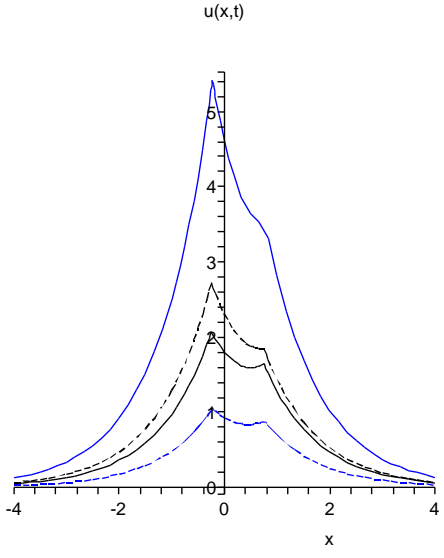

Figure 5: The two-peakon solution determined by (52). Solid line: $u(x, t)$; Dashed line: $v(x, t)$; Black: $t=-0.5$; Blue: $t=-1$.

where $A_{1}, A_{2}, B_{1}, B_{2}, B_{3}$, and $B_{4}$ are six integration constants. Let us consider a special case of choosing $A_{1}=B_{1}=B_{2}=B_{3}=1, A_{2}=4, B_{4}=0$. Then we have

$$
\left\{\begin{array}{l}
p_{1}=e^{-\frac{1}{3} t+\frac{1}{2} e^{-t-1}-2 e^{t-1}} \\
p_{2}=e^{-\frac{4}{3} t+\frac{1}{2} e^{-t-1}-2 e^{t-1}} \\
r_{1}=e^{\frac{1}{3} t-\frac{1}{2} e^{-t-1}+2 e^{t-1}} \\
r_{2}=4 e^{\frac{4}{3} t-\frac{1}{2} e^{-t-1}+2 e^{t-1}} \\
q_{1}=\frac{1}{2} e^{-t-1}+2 e^{t-1} \\
q_{2}=q_{1}-1
\end{array}\right.
$$

Figure 5 shows the dynamics of this two-peakon for the potentials $u(x, t)$ and $v(x, t)$ determined by (52).

Remark 4. It has been shown equations (37) and (42) share the same bi-Hamiltonian operators (but with different Hamiltonian functions). In fact, the bi-Hamiltonian operators (39) generate two hierarchies of equations. To see this, we define Lenard sequence recursively by

$$
J b_{-k}=K b_{-k+1}, \quad J b_{0}=0, \quad k \in \mathbb{Z}^{+},
$$

and the soliton hierarchy by

$$
m_{t_{-n}}=K b_{-n}, \quad n \in \mathbb{Z}^{+} .
$$

Let us take an initial value $b_{0}=(0,0)^{T}$. Then from $J b_{-1}=K b_{0}$, we may reach $b_{-1}=\frac{1}{2}(v, u)^{T}$ or $b_{-1}=\frac{1}{2}\left(v_{x},-u_{x}\right)^{T}$. For $b_{-1}=\frac{1}{2}(v, u)^{T}$, the first member $m_{t_{-1}}=K b_{-1}$ in the hierarchy (53) 
is just equation (37). While for $b_{-1}=\frac{1}{2}\left(v_{x},-u_{x}\right)^{T}$, the first member $m_{t_{-1}}=K b_{-1}$ is nothing but equation (42).

Remark 5. Although equations (37) and (42) share the same bi-Hamiltonian operators, their peakon dynamics are very different. In the single-peakon case, the peakon solution of (37) is in the type of traveling wave (see (41)), while the peakon solution of (42) is not, since the peak point does not change along with the time $t$ (see (45)). In the two-peakon case, the collision of the two-peakon of equation (37) is discussed in [28], while the two-peakon of equation (42) never collides since their positions are satisfied with $q_{2}(t)=q_{1}(t)-B_{1}$, where $B_{1}$ is a nonzero constant.

Example 4. The two-component integrable system proposed by Song, Qu, and Qiao [27]

Choosing $H=\frac{1}{2}\left(u-u_{x}\right)\left(v+v_{x}\right)$ casts equation (7) into

$$
\left\{\begin{array}{l}
m_{t}=\frac{1}{2}\left[m\left(u-u_{x}\right)\left(v+v_{x}\right)\right]_{x} \\
n_{t}=\frac{1}{2}\left[n\left(u-u_{x}\right)\left(v+v_{x}\right)\right]_{x} \\
m=u-u_{x x}, \quad n=v-v_{x x}
\end{array}\right.
$$

which is exactly the equation derived by Song, Qu, and Qiao [27]. This system possesses a bi-Hamiltonian structure [39]:

$$
\left(m_{t}, n_{t}\right)^{T}=J\left(\frac{\delta H_{2}}{\delta m}, \frac{\delta H_{2}}{\delta n}\right)^{T}=K\left(\frac{\delta H_{1}}{\delta m}, \frac{\delta H_{1}}{\delta n}\right)^{T}
$$

where

$$
\begin{aligned}
J & =\left(\begin{array}{cc}
0 & \partial^{2}+\partial \\
-\partial^{2}+\partial & 0
\end{array}\right), \quad K=\left(\begin{array}{cc}
\partial m \partial^{-1} m \partial & \partial m \partial^{-1} n \partial \\
\partial n \partial^{-1} m \partial & \partial n \partial^{-1} n \partial
\end{array}\right), \\
H_{1} & =\frac{1}{2} \int_{-\infty}^{+\infty} m\left(v+v_{x}\right) d x, \quad H_{2}=\frac{1}{4} \int_{-\infty}^{+\infty}\left(u-u_{x}\right)^{2}\left(v+v_{x}\right) n d x
\end{aligned}
$$

In the following, we want to derive the peakon solutions and discuss the peakon interactions for this system. It is easy to check that the one-peakon solution of (154) takes the same form as (41). In general, by direct calculations, we can obtain the $N$-peakon dynamical system of (54) as follows

$$
\left\{\begin{array}{l}
p_{j, t}=0 \\
r_{j, t}=0 \\
q_{j, t}=\frac{1}{6} p_{j} r_{j}+\frac{1}{2} \sum_{i, k=1}^{N} p_{i} r_{k}\left(\operatorname{sgn}\left(q_{j}-q_{i}\right)+1\right)\left(\operatorname{sgn}\left(q_{j}-q_{k}\right)-1\right) e^{-\left|q_{j}-q_{i}\right|-\left|q_{j}-q_{k}\right|} .
\end{array}\right.
$$


If $N=2$, then the two-peakon system reads as

$$
\left\{\begin{array}{l}
p_{1, t}=p_{2, t}=r_{1, t}=r_{2, t}=0 \\
q_{1, t}=-\frac{1}{3} p_{1} r_{1}+\frac{1}{2}\left[p_{1} r_{2}\left(\operatorname{sgn}\left(q_{1}-q_{2}\right)-1\right)-p_{2} r_{1}\left(\operatorname{sgn}\left(q_{1}-q_{2}\right)+1\right)\right] e^{-\left|q_{1}-q_{2}\right|} \\
q_{2, t}=-\frac{1}{3} p_{2} r_{2}+\frac{1}{2}\left[p_{1} r_{2}\left(\operatorname{sgn}\left(q_{1}-q_{2}\right)-1\right)-p_{2} r_{1}\left(\operatorname{sgn}\left(q_{1}-q_{2}\right)+1\right)\right] e^{-\left|q_{1}-q_{2}\right|}
\end{array}\right.
$$

From the first equation of (59), we know

$$
p_{1}=A_{1}, \quad p_{2}=A_{2}, \quad r_{1}=B_{1}, \quad r_{2}=B_{2},
$$

where $A_{1}, A_{2}, B_{1}$, and $B_{2}$ are four integration constants. If $A_{1} B_{1}=A_{2} B_{2}$, then we have

$$
\left\{\begin{array}{l}
q_{1}(t)=\left\{-\frac{1}{3} A_{1} B_{1}+\frac{1}{2}\left[A_{1} B_{2}\left(\operatorname{sgn}\left(C_{1}\right)-1\right)-A_{2} B_{1}\left(\operatorname{sgn}\left(C_{1}\right)+1\right)\right] e^{-\left|C_{1}\right|}\right\} t+\frac{C_{1}}{2}, \\
q_{2}(t)=q_{1}(t)-C_{1} .
\end{array}\right.
$$

If $A_{1} B_{1} \neq A_{2} B_{2}$, then we arrive at

$$
\left\{\begin{array}{l}
q_{1}(t)=-\frac{1}{3} A_{1} B_{1} t+\Gamma(t) \\
q_{2}(t)=-\frac{1}{3} A_{2} B_{2} t++\Gamma(t)
\end{array}\right.
$$

where

$\Gamma(t)=\frac{3\left(A_{1} B_{2}+A_{2} B_{1}\right)}{2\left|A_{1} B_{1}-A_{2} B_{2}\right|} \operatorname{sgn}(t)\left(e^{-\frac{1}{3}\left|\left(A_{1} B_{1}-A_{2} B_{2}\right) t\right|}-1\right)+\frac{3\left(A_{1} B_{2}-A_{2} B_{1}\right)}{2\left(A_{1} B_{1}-A_{2} B_{2}\right)} e^{-\frac{1}{3}\left|\left(A_{1} B_{1}-A_{2} B_{2}\right) t\right|}$.

In particular, taking $A_{1}=B_{1}=1, A_{2}=2$, and $B_{2}=5$ sends the two-peakon solution to the following form

$$
\left\{\begin{array}{l}
u(x, t)=e^{-\left|x-q_{1}(t)\right|}+2 e^{-\left|x-q_{2}(t)\right|}, \\
v(x, t)=e^{-\left|x-q_{1}(t)\right|}+5 e^{-\left|x-q_{2}(t)\right|}
\end{array}\right.
$$

where

$$
\left\{\begin{array}{l}
q_{1}(t)=-\frac{t}{3}+\frac{7}{6} \operatorname{sgn}(t)\left(e^{-3|t|}-1\right)-\frac{1}{2} e^{-3|t|} \\
q_{2}(t)=-\frac{10 t}{3}+\frac{7}{6} \operatorname{sgn}(t)\left(e^{-3|t|}-1\right)-\frac{1}{2} e^{-3|t|}
\end{array}\right.
$$

For the potential $u(x, t)$, the two-peakon collides at the moment $t=0$, since $q_{1}(0)=q_{2}(0)=0$. For $t<0$, the tall and fast peakon with the amplitude 2 and peak position $q_{2}$ chases after the short and slow peakon with the amplitude 1 and peak position $q_{1}$. At the moment of $t=0$, the two-peakon overlaps. After the collision $(t>0)$, the two-peakon separates, and the tall and fast peakon surpasses the short and slow one. Similarly, we may discuss the collision of the two-peakon for the potential $v(x, t)$. See Figures 6 and 7 for the two-peakon dynamics of the potentials $u(x, t)$ and $v(x, t)$. 


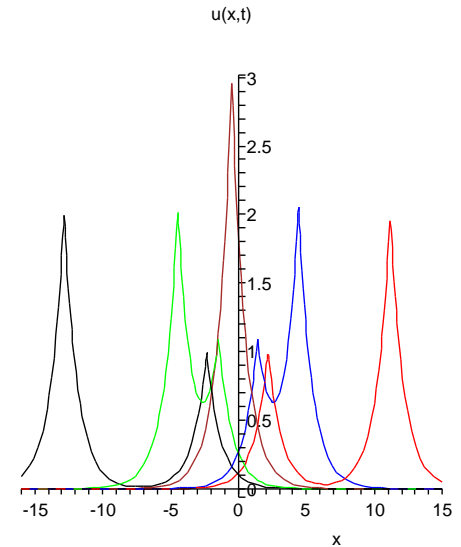

Figure 6: The two-peakon solution for the potential $u(x, t)$ given by (64). Red line: $t=-3$; Blue line: $t=-1$; Brown line: $t=0$ (collision); Green line: $t=1$; Black line: $t=3.5$.

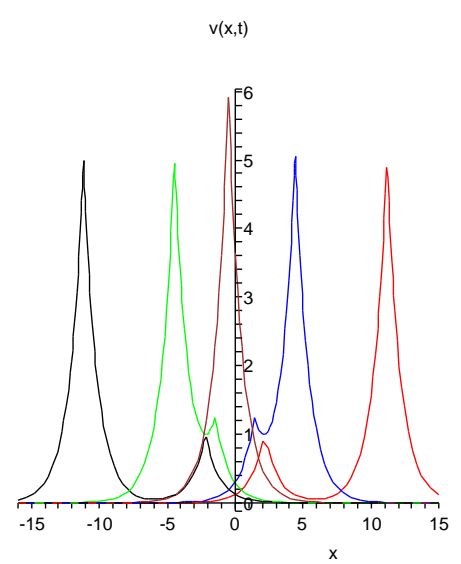

Figure 7: The two-peakon solution for the potential $v(x, t)$ given by (64). Red line: $t=-3$; Blue line: $t=-1$; Brown line: $t=0$ (collision); Green line: $t=1$; Black line: $t=3$.

\section{A proof for the bi-Hamiltonian property}

In this section, we will supply a proof for the bi-Hamiltonian property in each example presented in the above section. Let us introduce the following basic operators

$$
\begin{aligned}
J_{1} & =\left(\begin{array}{cc}
0 & -1 \\
1 & 0
\end{array}\right), \quad J_{2}=\left(\begin{array}{cc}
0 & \partial \\
\partial & 0
\end{array}\right), \quad J_{3}=\left(\begin{array}{cc}
0 & \partial^{2} \\
-\partial^{2} & 0
\end{array}\right), \\
K_{1} & =\left(\begin{array}{cc}
-m \partial^{-1} m & m \partial^{-1} n \\
n \partial^{-1} m & -n \partial^{-1} n
\end{array}\right), \quad K_{2}=\left(\begin{array}{cc}
\partial m \partial^{-1} m \partial & \partial m \partial^{-1} n \partial \\
\partial n \partial^{-1} m \partial & \partial n \partial^{-1} n \partial
\end{array}\right) .
\end{aligned}
$$

Lemma 1 All the above operators are Hamiltonian operators.

Proof It is obvious that $J_{1}, J_{2}$ and $J_{3}$ are Hamiltonian operators since they are skewsymmetric operators with constant-coefficient. It is easy to check $K_{1}$ and $K_{2}$ are skew-symmetric. We need to prove that both $K_{1}$ and $K_{2}$ satisfy the Jacobi identities

$$
\begin{aligned}
& \left\langle\alpha, K_{1}^{\prime}\left[K_{1} \beta\right] \gamma\right\rangle+\operatorname{cycle}(\alpha, \beta, \gamma)=0, \\
& \left\langle\alpha, K_{2}^{\prime}\left[K_{2} \beta\right] \gamma\right\rangle+\operatorname{cycle}(\alpha, \beta, \gamma)=0,
\end{aligned}
$$

where $\alpha=\left(\alpha_{1}, \alpha_{2}\right)^{T}, \beta=\left(\beta_{1}, \beta_{2}\right)^{T}, \gamma=\left(\gamma_{1}, \gamma_{2}\right)^{T}$ are arbitrary vector functions, the symbol $\operatorname{cycle}(\alpha, \beta, \gamma)$ means the cyclic permutation of $\alpha, \beta, \gamma$, and the prime-sign means the Gâteaux derivative of an operator $F$ on $q$ in the direction $\sigma$ defined as [18]

$$
F^{\prime}[\sigma]=F^{\prime}(q)[\sigma]=\left.\frac{\partial}{\partial \epsilon}\right|_{\epsilon=0} F(q+\epsilon \sigma) .
$$


Let us first prove the Jacobi identity (68). For brevity, we introduce the notations

$$
A=\partial^{-1}\left(m \alpha_{1}-n \alpha_{2}\right), \quad B=\partial^{-1}\left(m \beta_{1}-n \beta_{2}\right), \quad C=\partial^{-1}\left(m \gamma_{1}-n \gamma_{2}\right) .
$$

Direct calculations give rise to

$$
\left\langle\alpha, K_{1}^{\prime}\left[K_{1} \beta\right] \gamma\right\rangle=\int_{-\infty}^{+\infty}\left[\left(\alpha_{1} m B+\alpha_{2} n B\right) C+\left(\alpha_{1} m-\alpha_{2} n\right) \partial^{-1}\left(\gamma_{1} m B+\gamma_{2} n B\right)\right] d x .
$$

Integrating (72) by parts, we obtain

$$
\begin{aligned}
\left\langle\alpha, K_{1}^{\prime}\left[K_{1} \beta\right] \gamma\right\rangle & =\int_{-\infty}^{+\infty}\left[\left(\alpha_{1} m B+\alpha_{2} n B\right) C-\left(\gamma_{1} m B+\gamma_{2} n B\right) \partial^{-1}\left(\alpha_{1} m-\alpha_{2} n\right)\right] d x \\
& =\int_{-\infty}^{+\infty}\left[\left(\alpha_{1} m B+\alpha_{2} n B\right) C-\left(\gamma_{1} m B+\gamma_{2} n B\right) A\right] d x \\
& =\int_{-\infty}^{+\infty}\left[\left(\alpha_{1} m+\alpha_{2} n\right) B C-\left(\gamma_{1} m+\gamma_{2} n\right) B A\right] d x
\end{aligned}
$$

Thus

$$
\begin{aligned}
& \left\langle\alpha, K_{1}^{\prime}\left[K_{1} \beta\right] \gamma\right\rangle+\operatorname{cycle}(\alpha, \beta, \gamma) \\
= & \int_{-\infty}^{+\infty}\left[\left(\alpha_{1} m+\alpha_{2} n\right) B C-\left(\gamma_{1} m+\gamma_{2} n\right) B A\right] d x \\
& +\int_{-\infty}^{+\infty}\left[\left(\beta_{1} m+\beta_{2} n\right) C A-\left(\alpha_{1} m+\alpha_{2} n\right) C B\right] d x \\
& +\int_{-\infty}^{+\infty}\left[\left(\gamma_{1} m+\gamma_{2} n\right) A B-\left(\beta_{1} m+\beta_{2} n\right) A C\right] d x
\end{aligned}
$$$$
=0 \text {. }
$$

Now we turn to the proof of Jacobi identity (69). Let us set

$$
\tilde{A}=\partial^{-1}\left(m \alpha_{1, x}+n \alpha_{2, x}\right), \quad \tilde{B}=\partial^{-1}\left(m \beta_{1, x}+n \beta_{2, x}\right), \quad \tilde{C}=\partial^{-1}\left(m \gamma_{1, x}+n \gamma_{2, x}\right) .
$$

With the similar calculations as (72) and (73), we arrive at

$$
\left\langle\alpha, K_{2}^{\prime}\left[K_{2} \beta\right] \gamma\right\rangle=\int_{-\infty}^{+\infty}\left[\left(\gamma_{1, x} m_{x}+\gamma_{2, x} n_{x}\right) \tilde{B} \tilde{A}-\left(\alpha_{1, x} m_{x}+\alpha_{2, x} n_{x}\right) \tilde{B} \tilde{C}+\tilde{C}_{x} \tilde{B}_{x} \tilde{A}-\tilde{A}_{x} \tilde{B_{x}} \tilde{C}\right] d x
$$

Then it follows that

$$
\begin{aligned}
& \left\langle\alpha, K_{2}^{\prime}\left[K_{2} \beta\right] \gamma\right\rangle+\operatorname{cycle}(\alpha, \beta, \gamma) \\
= & \int_{-\infty}^{+\infty}\left[\left(\gamma_{1, x} m_{x}+\gamma_{2, x} n_{x}\right) \tilde{B} \tilde{A}-\left(\alpha_{1, x} m_{x}+\alpha_{2, x} n_{x}\right) \tilde{B} \tilde{C}+\tilde{C}_{x} \tilde{B_{x}} \tilde{A}-\tilde{A_{x}} \tilde{B_{x}} \tilde{C}\right] d x \\
& +\int_{-\infty}^{+\infty}\left[\left(\alpha_{1, x} m_{x}+\alpha_{2, x} n_{x}\right) \tilde{C} \tilde{B}-\left(\beta_{1, x} m_{x}+\beta_{2, x} n_{x}\right) \tilde{C} \tilde{A}+\tilde{A}_{x} \tilde{C_{x}} \tilde{B}-\tilde{B}_{x} \tilde{C_{x}} \tilde{A}\right] d x \\
& +\int_{-\infty}^{+\infty}\left[\left(\beta_{1, x} m_{x}+\beta_{2, x} n_{x}\right) \tilde{A} \tilde{C}-\left(\gamma_{1, x} m_{x}+\gamma_{2, x} n_{x}\right) \tilde{A} \tilde{B}+\tilde{B}_{x} \tilde{A_{x}} \tilde{C}-\tilde{C}_{x} \tilde{A_{x}} \tilde{B}\right] d x \\
= & 0 .
\end{aligned}
$$


The proof of lemma 1 is finished.

Lemma 2 The following relations hold

$$
\begin{aligned}
\left\langle\alpha, J_{1}^{\prime}\left[J_{2} \beta\right] \gamma\right\rangle+\left\langle\alpha, J_{2}^{\prime}\left[J_{1} \beta\right] \gamma\right\rangle+\operatorname{cycle}(\alpha, \beta, \gamma) & =0 \\
\left\langle\alpha, J_{1}^{\prime}\left[J_{3} \beta\right] \gamma\right\rangle+\left\langle\alpha, J_{3}^{\prime}\left[J_{1} \beta\right] \gamma\right\rangle+\operatorname{cycle}(\alpha, \beta, \gamma) & =0 \\
\left\langle\alpha, J_{2}^{\prime}\left[J_{3} \beta\right] \gamma\right\rangle+\left\langle\alpha, J_{3}^{\prime}\left[J_{2} \beta\right] \gamma\right\rangle+\operatorname{cycle}(\alpha, \beta, \gamma) & =0 \\
\left\langle\alpha, J_{1}^{\prime}\left[K_{1} \beta\right] \gamma\right\rangle+\left\langle\alpha, K_{1}^{\prime}\left[J_{1} \beta\right] \gamma\right\rangle+\operatorname{cycle}(\alpha, \beta, \gamma) & =0 \\
\left\langle\alpha, J_{2}^{\prime}\left[K_{1} \beta\right] \gamma\right\rangle+\left\langle\alpha, K_{1}^{\prime}\left[J_{2} \beta\right] \gamma\right\rangle+\operatorname{cycle}(\alpha, \beta, \gamma) & =0 \\
\left\langle\alpha, J_{2}^{\prime}\left[K_{2} \beta\right] \gamma\right\rangle+\left\langle\alpha, K_{2}^{\prime}\left[J_{2} \beta\right] \gamma\right\rangle+\operatorname{cycle}(\alpha, \beta, \gamma) & =0 \\
\left\langle\alpha, J_{3}^{\prime}\left[K_{2} \beta\right] \gamma\right\rangle+\left\langle\alpha, K_{2}^{\prime}\left[J_{3} \beta\right] \gamma\right\rangle+\operatorname{cycle}(\alpha, \beta, \gamma) & =0 \\
\left\langle\alpha, K_{1}^{\prime}\left[K_{2} \beta\right] \gamma\right\rangle+\left\langle\alpha, K_{2}^{\prime}\left[K_{1} \beta\right] \gamma\right\rangle+\operatorname{cycle}(\alpha, \beta, \gamma) & =0 .
\end{aligned}
$$

Proof It is clearly formulas (78)-(80) hold since $J_{1}, J_{2}$ and $J_{3}$ are constant-coefficient operators. For (81), we have $\left\langle\alpha, J_{1}^{\prime}\left[K_{1} \beta\right] \gamma\right\rangle=0$, and

$$
\left\langle\alpha, K_{1}^{\prime}\left[J_{1} \beta\right] \gamma\right\rangle=\int_{-\infty}^{+\infty}\left[\left(\alpha_{1} \beta_{2}+\alpha_{2} \beta_{1}\right) C-\left(\beta_{1} \gamma_{2}+\beta_{2} \gamma_{1}\right) A\right] d x .
$$

Thus the left hand side (LHS) of (81) becomes

$$
\begin{aligned}
& \int_{-\infty}^{+\infty}\left[\left(\alpha_{1} \beta_{2}+\alpha_{2} \beta_{1}\right) C-\left(\beta_{1} \gamma_{2}+\beta_{2} \gamma_{1}\right) A\right] d x+\int_{-\infty}^{+\infty}\left[\left(\beta_{1} \gamma_{2}+\beta_{2} \gamma_{1}\right) A-\left(\gamma_{1} \alpha_{2}+\gamma_{2} \alpha_{1}\right) B\right] d x \\
& +\int_{-\infty}^{+\infty}\left[\left(\gamma_{1} \alpha_{2}+\gamma_{2} \alpha_{1}\right) B-\left(\alpha_{1} \beta_{2}+\alpha_{2} \beta_{1}\right) C\right] d x \\
= & 0 .
\end{aligned}
$$

Similarly, by direct calculations, the LHS of (82) becomes

$$
\begin{aligned}
& \int_{-\infty}^{+\infty}\left[\left(\beta_{1, x} \alpha_{2}-\beta_{2, x} \alpha_{1}\right) C-\left(\beta_{1, x} \gamma_{2}-\beta_{2, x} \gamma_{1}\right) A\right] d x \\
+ & \int_{-\infty}^{+\infty}\left[\left(\gamma_{1, x} \beta_{2}-\gamma_{2, x} \beta_{1}\right) A-\left(\gamma_{1, x} \alpha_{2}-\gamma_{2, x} \alpha_{1}\right) B\right] d x \\
+ & \int_{-\infty}^{+\infty}\left[\left(\alpha_{1, x} \gamma_{2}-\alpha_{2, x} \gamma_{1}\right) B-\left(\alpha_{1, x} \beta_{2}-\alpha_{2, x} \beta_{1}\right) C\right] d x \\
= & \int_{-\infty}^{+\infty}\left[\left(\beta_{1} \alpha_{2}-\beta_{2} \alpha_{1}\right)_{x} C-\left(\beta_{1} \gamma_{2}-\beta_{2} \gamma_{1}\right)_{x} A-\left(\gamma_{1} \alpha_{2}-\gamma_{2} \alpha_{1}\right)_{x} B\right] d x \\
= & -\int_{-\infty}^{+\infty}\left[\left(\beta_{1} \alpha_{2}-\beta_{2} \alpha_{1}\right) C_{x}-\left(\beta_{1} \gamma_{2}-\beta_{2} \gamma_{1}\right) A_{x}-\left(\gamma_{1} \alpha_{2}-\gamma_{2} \alpha_{1}\right) B_{x}\right] d x \\
= & -\int_{-\infty}^{+\infty}\left[\left(\beta_{1} \alpha_{2}-\beta_{2} \alpha_{1}\right)\left(m \gamma_{1}-n \gamma_{2}\right)-\left(\beta_{1} \gamma_{2}-\beta_{2} \gamma_{1}\right)\left(m \alpha_{1}-n \alpha_{2}\right)-\left(\gamma_{1} \alpha_{2}-\gamma_{2} \alpha_{1}\right)\left(m \beta_{1}-n \beta_{2}\right)\right] d x \\
= & 0 .
\end{aligned}
$$


The LHS of (83) is equal to

$$
\begin{aligned}
& -\int_{-\infty}^{+\infty}\left[\left(\alpha_{1, x} \beta_{2, x}+\alpha_{2, x} \beta_{1, x}\right) \tilde{C}-\left(\beta_{2, x} \gamma_{1, x}+\beta_{1, x} \gamma_{2, x}\right) \tilde{A}\right] d x \\
& -\int_{-\infty}^{+\infty}\left[\left(\beta_{1, x} \gamma_{2, x}+\beta_{2, x} \gamma_{1, x}\right) \tilde{A}-\left(\gamma_{2, x} \alpha_{1, x}+\gamma_{1, x} \alpha_{2, x}\right) \tilde{B}\right] d x \\
& -\int_{-\infty}^{+\infty}\left[\left(\gamma_{1, x} \alpha_{2, x}+\gamma_{2, x} \alpha_{1, x}\right) \tilde{B}-\left(\alpha_{2, x} \beta_{1, x}+\alpha_{1, x} \beta_{2, x}\right) \tilde{C}\right] d x \\
= & 0 .
\end{aligned}
$$

The LHS of (84) is equal to

$$
\begin{aligned}
& -\int_{-\infty}^{+\infty}\left[\left(\alpha_{1, x} \beta_{2, x x}-\alpha_{2, x} \beta_{1, x x}\right) \tilde{C}-\left(\beta_{2, x x} \gamma_{1, x}-\beta_{1, x x} \gamma_{2, x}\right) \tilde{A}\right] d x \\
& -\int_{-\infty}^{+\infty}\left[\left(\beta_{1, x} \gamma_{2, x x}-\beta_{2, x} \gamma_{1, x x}\right) \tilde{A}-\left(\gamma_{2, x x} \alpha_{1, x}-\gamma_{1, x x} \alpha_{2, x}\right) \tilde{B}\right] d x \\
& -\int_{-\infty}^{+\infty}\left[\left(\gamma_{1, x} \alpha_{2, x x}-\gamma_{2, x} \alpha_{1, x x}\right) \tilde{B}-\left(\alpha_{2, x x} \beta_{1, x}-\alpha_{1, x x} \beta_{2, x}\right) \tilde{C}\right] d x \\
= & -\int_{-\infty}^{+\infty}\left[\left(\beta_{1, x} \gamma_{2, x}-\beta_{2, x} \gamma_{1, x}\right)_{x} \tilde{A}+\left(\gamma_{1, x} \alpha_{2, x}-\gamma_{2, x} \alpha_{1, x}\right)_{x} \tilde{B}+\left(\alpha_{1, x} \beta_{2, x}-\alpha_{2, x} \beta_{1, x}\right){ }_{x} \tilde{C}\right] d x \\
= & \int_{-\infty}^{+\infty}\left[\left(\beta_{1, x} \gamma_{2, x}-\beta_{2, x} \gamma_{1, x}\right) \tilde{A}_{x}+\left(\gamma_{1, x} \alpha_{2, x}-\gamma_{2, x} \alpha_{1, x}\right) \tilde{B}_{x}+\left(\alpha_{1, x} \beta_{2, x}-\alpha_{2, x} \beta_{1, x}\right) \tilde{C}_{x}\right] d x \\
= & \int_{-\infty}^{+\infty}\left[\left(\beta_{1, x} \gamma_{2, x}-\beta_{2, x} \gamma_{1, x}\right)\left(m \alpha_{1, x}+n \alpha_{2, x}\right)+\left(\gamma_{1, x} \alpha_{2, x}-\gamma_{2, x} \alpha_{1, x}\right)\left(m \beta_{1, x}+n \beta_{2, x}\right)\right. \\
= & 0 .
\end{aligned}
$$

For the LHS of (85), we have

$$
\begin{aligned}
& \left\langle\alpha, K_{1}^{\prime}\left[K_{2} \beta\right] \gamma\right\rangle=\int_{-\infty}^{+\infty}\left[\left(m \alpha_{1, x}-n \alpha_{2, x}\right) C \tilde{B}-\left(m \gamma_{1, x}-n \gamma_{2, x}\right) A \tilde{B}\right] d x \\
& \left\langle\alpha, K_{2}^{\prime}\left[K_{1} \beta\right] \gamma\right\rangle=\int_{-\infty}^{+\infty}\left[\left(m \alpha_{1, x}-n \alpha_{2, x}\right) B \tilde{C}-\left(m \gamma_{1, x}-n \gamma_{2, x}\right) B \tilde{A}\right] d x
\end{aligned}
$$

Hence the LHS of (85) reads as

$$
\begin{aligned}
& \int_{-\infty}^{+\infty}\left[\left(m \alpha_{1, x}-n \alpha_{2, x}\right)(C \tilde{B}+B \tilde{C})-\left(m \gamma_{1, x}-n \gamma_{2, x}\right)(A \tilde{B}+B \tilde{A})\right] d x \\
+ & \int_{-\infty}^{+\infty}\left[\left(m \beta_{1, x}-n \beta_{2, x}\right)(A \tilde{C}+C \tilde{A})-\left(m \alpha_{1, x}-n \alpha_{2, x}\right)(B \tilde{C}+C \tilde{B})\right] d x \\
+ & \int_{-\infty}^{+\infty}\left[\left(m \gamma_{1, x}-n \gamma_{2, x}\right)(B \tilde{A}+A \tilde{B})-\left(m \beta_{1, x}-n \beta_{2, x}\right)(C \tilde{A}+A \tilde{C})\right] d x \\
= & 0 .
\end{aligned}
$$


This completes the proof of lemma 2 .

Lemma 2 implies that $J_{1}+J_{2}, J_{1}+J_{3}, J_{1}+K_{1}, J_{2}+J_{3}, J_{2}+K_{1}, J_{2}+K_{2}, J_{3}+K_{2}$ and $K_{1}+K_{2}$ are Hamiltonian operators. But we should notice that $J_{1}+K_{2}$ and $J_{3}+K_{1}$ are not Hamiltonian operators. In fact, we have

Lemma 3 The following two relations hold

$$
\begin{aligned}
& \left\langle\alpha, J_{1}^{\prime}\left[K_{2} \beta\right] \gamma\right\rangle+\left\langle\alpha, K_{2}^{\prime}\left[J_{1} \beta\right] \gamma\right\rangle+\operatorname{cycle}(\alpha, \beta, \gamma) \\
= & \int_{-\infty}^{+\infty}\left[\left(\beta_{1} \gamma_{2}-\beta_{2} \gamma_{1}\right)_{x} \tilde{A}+\left(\alpha_{2} \gamma_{1}-\alpha_{1} \gamma_{2}\right)_{x} \tilde{B}+\left(\alpha_{1} \beta_{2}-\alpha_{2} \beta_{1}\right)_{x} \tilde{C}\right] d x, \\
& \left\langle\alpha, J_{3}^{\prime}\left[K_{1} \beta\right] \gamma\right\rangle+\left\langle\alpha, K_{1}^{\prime}\left[J_{3} \beta\right] \gamma\right\rangle+\operatorname{cycle}(\alpha, \beta, \gamma) \\
= & \int_{-\infty}^{+\infty}\left[\left(\beta_{1, x} \gamma_{2}-\beta_{1} \gamma_{2, x}+\beta_{2, x} \gamma_{1}-\beta_{2} \gamma_{1, x}\right)_{x} A+\left(\gamma_{1, x} \alpha_{2}-\gamma_{1} \alpha_{2, x}+\gamma_{2, x} \alpha_{1}-\gamma_{2} \alpha_{1, x}\right)_{x} B\right. \\
& \left.\quad+\left(\alpha_{1, x} \beta_{2}-\alpha_{1} \beta_{2, x}+\alpha_{2, x} \beta_{1}-\alpha_{2} \beta_{1, x}\right)_{x} C\right] d x .
\end{aligned}
$$

Proof Direct calculations yield that

$$
\begin{aligned}
&\left\langle\alpha, J_{1}^{\prime}\left[K_{2} \beta\right] \gamma\right\rangle+\left\langle\alpha, K_{2}^{\prime}\left[J_{1} \beta\right] \gamma\right\rangle+\operatorname{cycle}(\alpha, \beta, \gamma) \\
&=-\int_{-\infty}^{+\infty}\left[\left(\beta_{1} \alpha_{2, x}-\beta_{2} \alpha_{1, x}\right) \tilde{C}-\left(\beta_{1} \gamma_{2, x}-\beta_{2} \gamma_{1, x}\right) \tilde{A}\right] d x \\
&-\int_{-\infty}^{+\infty}\left[\left(\gamma_{1} \beta_{2, x}-\gamma_{2} \beta_{1, x}\right) \tilde{A}-\left(\gamma_{1} \alpha_{2, x}-\gamma_{2} \alpha_{1, x}\right) \tilde{B}\right] d x \\
&-\int_{-\infty}^{+\infty}\left[\left(\alpha_{1} \gamma_{2, x}-\alpha_{2} \gamma_{1, x}\right) \tilde{B}-\left(\alpha_{1} \beta_{2, x}-\alpha_{2} \beta_{1, x}\right) \tilde{C}\right] d x \\
&= \int_{-\infty}^{+\infty}\left[\left(\beta_{1} \gamma_{2}-\beta_{2} \gamma_{1}\right)_{x} \tilde{A}+\left(\alpha_{2} \gamma_{1}-\alpha_{1} \gamma_{2}\right)_{x} \tilde{B}+\left(\alpha_{1} \beta_{2}-\alpha_{2} \beta_{1}\right)_{x} \tilde{C}\right] d x,
\end{aligned}
$$

and

$$
\begin{aligned}
& \left\langle\alpha, J_{3}^{\prime}\left[K_{1} \beta\right] \gamma\right\rangle+\left\langle\alpha, K_{1}^{\prime}\left[J_{3} \beta\right] \gamma\right\rangle+\operatorname{cycle}(\alpha, \beta, \gamma) \\
= & -\int_{-\infty}^{+\infty}\left[\left(\alpha_{1} \beta_{2, x x}+\alpha_{2} \beta_{1, x x}\right) C-\left(\beta_{1, x x} \gamma_{2}+\beta_{2, x x} \gamma_{1}\right) A\right] d x \\
& -\int_{-\infty}^{+\infty}\left[\left(\beta_{1} \gamma_{2, x x}+\beta_{2} \gamma_{1, x x}\right) A-\left(\gamma_{1, x x} \alpha_{2}+\gamma_{2, x x} \alpha_{1}\right) B\right] d x \\
& -\int_{-\infty}^{+\infty}\left[\left(\gamma_{1} \alpha_{2, x x}+\gamma_{2} \alpha_{1, x x}\right) B-\left(\alpha_{1, x x} \beta_{2}+\alpha_{2, x x} \beta_{1}\right) C\right] d x \\
= & \int_{-\infty}^{+\infty}\left[\left(\beta_{1, x} \gamma_{2}-\beta_{1} \gamma_{2, x}+\beta_{2, x} \gamma_{1}-\beta_{2} \gamma_{1, x}\right)_{x} A+\left(\gamma_{1, x} \alpha_{2}-\gamma_{1} \alpha_{2, x}+\gamma_{2, x} \alpha_{1}-\gamma_{2} \alpha_{1, x}\right)_{x} B\right. \\
& \left.\quad+\left(\alpha_{1, x} \beta_{2}-\alpha_{1} \beta_{2, x}+\alpha_{2, x} \beta_{1}-\alpha_{2} \beta_{1, x}\right)_{x} C\right] d x .
\end{aligned}
$$

This finishes the proof of lemma 3 . 
Lemma 4 The following Jacobi identity holds

$$
\left\langle\alpha, J_{1}^{\prime}\left[K_{2} \beta\right] \gamma\right\rangle+\left\langle\alpha, K_{2}^{\prime}\left[J_{1} \beta\right] \gamma\right\rangle+\left\langle\alpha, J_{3}^{\prime}\left[K_{1} \beta\right] \gamma\right\rangle+\left\langle\alpha, K_{1}^{\prime}\left[J_{3} \beta\right] \gamma\right\rangle+\operatorname{cycle}(\alpha, \beta, \gamma)=0 .
$$

Proof By virtue of lemma 3 and integration by parts, we arrive at

$$
\begin{aligned}
& \left\langle\alpha, J_{1}^{\prime}\left[K_{2} \beta\right] \gamma\right\rangle+\left\langle\alpha, K_{2}^{\prime}\left[J_{1} \beta\right] \gamma\right\rangle+\left\langle\alpha, J_{3}^{\prime}\left[K_{1} \beta\right] \gamma\right\rangle+\left\langle\alpha, K_{1}^{\prime}\left[J_{3} \beta\right] \gamma\right\rangle+\operatorname{cycle}(\alpha, \beta, \gamma) \\
& =\int_{-\infty}^{+\infty}\left[\left(\beta_{1} \gamma_{2}-\beta_{2} \gamma_{1}\right)_{x} \tilde{A}+\left(\alpha_{2} \gamma_{1}-\alpha_{1} \gamma_{2}\right)_{x} \tilde{B}+\left(\alpha_{1} \beta_{2}-\alpha_{2} \beta_{1}\right)_{x} \tilde{C}\right. \\
& +\left(\beta_{1, x} \gamma_{2}-\beta_{1} \gamma_{2, x}+\beta_{2, x} \gamma_{1}-\beta_{2} \gamma_{1, x}\right)_{x} A+\left(\gamma_{1, x} \alpha_{2}-\gamma_{1} \alpha_{2, x}+\gamma_{2, x} \alpha_{1}-\gamma_{2} \alpha_{1, x}\right)_{x} B \\
& \left.+\left(\alpha_{1, x} \beta_{2}-\alpha_{1} \beta_{2, x}+\alpha_{2, x} \beta_{1}-\alpha_{2} \beta_{1, x}\right)_{x} C\right] d x \\
& =-\int_{-\infty}^{+\infty}\left[\left(\beta_{1} \gamma_{2}-\beta_{2} \gamma_{1}\right) \tilde{A}_{x}+\left(\alpha_{2} \gamma_{1}-\alpha_{1} \gamma_{2}\right) \tilde{B}_{x}+\left(\alpha_{1} \beta_{2}-\alpha_{2} \beta_{1}\right) \tilde{C}_{x}\right. \\
& +\left(\beta_{1, x} \gamma_{2}-\beta_{1} \gamma_{2, x}+\beta_{2, x} \gamma_{1}-\beta_{2} \gamma_{1, x}\right) A_{x}+\left(\gamma_{1, x} \alpha_{2}-\gamma_{1} \alpha_{2, x}+\gamma_{2, x} \alpha_{1}-\gamma_{2} \alpha_{1, x}\right) B_{x} \\
& \left.+\left(\alpha_{1, x} \beta_{2}-\alpha_{1} \beta_{2, x}+\alpha_{2, x} \beta_{1}-\alpha_{2} \beta_{1, x}\right) C_{x}\right] d x \\
& =-\int_{-\infty}^{+\infty}\left[\left(\beta_{1} \gamma_{2}-\beta_{2} \gamma_{1}\right)\left(m \alpha_{1, x}+n \alpha_{2, x}\right)+\left(\alpha_{2} \gamma_{1}-\alpha_{1} \gamma_{2}\right)\left(m \beta_{1, x}+n \beta_{2, x}\right)\right. \\
& +\left(\alpha_{1} \beta_{2}-\alpha_{2} \beta_{1}\right)\left(m \gamma_{1, x}+n \gamma_{2, x}\right)+\left(\beta_{1, x} \gamma_{2}-\beta_{1} \gamma_{2, x}+\beta_{2, x} \gamma_{1}-\beta_{2} \gamma_{1, x}\right)\left(m \alpha_{1}-n \alpha_{2}\right) \\
& +\left(\gamma_{1, x} \alpha_{2}-\gamma_{1} \alpha_{2, x}+\gamma_{2, x} \alpha_{1}-\gamma_{2} \alpha_{1, x}\right)\left(m \beta_{1}-n \beta_{2}\right) \\
& \left.+\left(\alpha_{1, x} \beta_{2}-\alpha_{1} \beta_{2, x}+\alpha_{2, x} \beta_{1}-\alpha_{2} \beta_{1, x}\right)\left(m \gamma_{1}-n \gamma_{2}\right)\right] d x
\end{aligned}
$$

$=0$.

The proof of lemma 4 is finished.

Based on the above lemmas, we finally obtain

Proposition 1 Let $c_{j}, 1 \leq j \leq 5$, be arbitrary constants. For any $c_{1} c_{5}=c_{3} c_{4}$, we can conclude that $J=c_{1} J_{1}+c_{2} J_{2}+c_{3} J_{3}+c_{4} K_{1}+c_{5} K_{2}$ is a Hamiltonian operator.

Proof We need to verify the Jacobi identity

$$
\left\langle\alpha, J^{\prime}[J \beta] \gamma\right\rangle+\operatorname{cycle}(\alpha, \beta, \gamma)=0
$$


In fact, we have

$$
\begin{aligned}
& \left\langle\alpha, J^{\prime}[J \beta] \gamma\right\rangle+\operatorname{cycle}(\alpha, \beta, \gamma) \\
= & c_{1}^{2}\left\langle\alpha, J_{1}^{\prime}\left[J_{1} \beta\right] \gamma\right\rangle+c_{2}^{2}\left\langle\alpha, J_{2}^{\prime}\left[J_{2} \beta\right] \gamma\right\rangle+c_{3}^{2}\left\langle\alpha, J_{3}^{\prime}\left[J_{3} \beta\right] \gamma\right\rangle+c_{4}^{2}\left\langle\alpha, K_{1}^{\prime}\left[K_{1} \beta\right] \gamma\right\rangle+c_{5}^{2}\left\langle\alpha, K_{2}^{\prime}\left[K_{2} \beta\right] \gamma\right\rangle \\
& +c_{1} c_{2}\left(\left\langle\alpha, J_{1}^{\prime}\left[J_{2} \beta\right] \gamma\right\rangle+\left\langle\alpha, J_{2}^{\prime}\left[J_{1} \beta\right] \gamma\right\rangle\right)+c_{1} c_{3}\left(\left\langle\alpha, J_{1}^{\prime}\left[J_{3} \beta\right] \gamma\right\rangle+\left\langle\alpha, J_{3}^{\prime}\left[J_{1} \beta\right] \gamma\right\rangle\right) \\
& +c_{1} c_{4}\left(\left\langle\alpha, J_{1}^{\prime}\left[K_{1} \beta\right] \gamma\right\rangle+\left\langle\alpha, K_{1}^{\prime}\left[J_{1} \beta\right] \gamma\right\rangle\right)+c_{1} c_{5}\left(\left\langle\alpha, J_{1}^{\prime}\left[K_{2} \beta\right] \gamma\right\rangle+\left\langle\alpha, K_{2}^{\prime}\left[J_{1} \beta\right] \gamma\right\rangle\right) \\
& +c_{2} c_{3}\left(\left\langle\alpha, J_{2}^{\prime}\left[J_{3} \beta\right] \gamma\right\rangle+\left\langle\alpha, J_{3}^{\prime}\left[J_{2} \beta\right] \gamma\right\rangle\right)+c_{2} c_{4}\left(\left\langle\alpha, J_{2}^{\prime}\left[K_{1} \beta\right] \gamma\right\rangle+\left\langle\alpha, K_{1}^{\prime}\left[J_{2} \beta\right] \gamma\right\rangle\right) \\
& +c_{2} c_{5}\left(\left\langle\alpha, J_{2}^{\prime}\left[K_{2} \beta\right] \gamma\right\rangle+\left\langle\alpha, K_{2}^{\prime}\left[J_{2} \beta\right] \gamma\right\rangle\right)+c_{3} c_{4}\left(\left\langle\alpha, J_{3}^{\prime}\left[K_{1} \beta\right] \gamma\right\rangle+\left\langle\alpha, K_{1}^{\prime}\left[J_{3} \beta\right] \gamma\right\rangle\right) \\
& +c_{3} c_{5}\left(\left\langle\alpha, J_{3}^{\prime}\left[K_{2} \beta\right] \gamma\right\rangle+\left\langle\alpha, K_{2}^{\prime}\left[J_{3} \beta\right] \gamma\right\rangle\right)+c_{4} c_{5}\left(\left\langle\alpha, K_{1}^{\prime}\left[K_{2} \beta\right] \gamma\right\rangle+\left\langle\alpha, K_{2}^{\prime}\left[K_{1} \beta\right] \gamma\right\rangle\right) \\
& +c y c l e(\alpha, \beta, \gamma) \\
= & c_{1} c_{5}\left(\left\langle\alpha, J_{1}^{\prime}\left[K_{2} \beta\right] \gamma\right\rangle+\left\langle\alpha, K_{2}^{\prime}\left[J_{1} \beta\right] \gamma\right\rangle\right)+c_{3} c_{4}\left(\left\langle\alpha, J_{3}^{\prime}\left[K_{1} \beta\right] \gamma\right\rangle+\left\langle\alpha, K_{1}^{\prime}\left[J_{3} \beta\right] \gamma\right\rangle\right)+\operatorname{cycle}(\alpha, \beta, \gamma) \\
= & 0,
\end{aligned}
$$

where the last identity holds because of $c_{1} c_{5}=c_{3} c_{4}$ and lemma 4 . This completes the proof of the proposition.

Recall that a pair of Hamiltonian operators $J$ and $K$ is called compatible, if $J+K$ is Hamiltonian. From the above proposition, we immediately arrive at

Corollary 1 The case of $c_{1}=-c_{2}=c_{4}=1$ and $c_{3}=c_{5}=0$ leads to the compatibility of the Hamiltonian operators (27).

Corollary 2 The case of $c_{1}=c_{3}=c_{4}=c_{5}=1$ and $c_{2}=0$ leads to the compatibility of the Hamiltonian operators (39).

Corollary 3 The case of $c_{2}=c_{3}=c_{5}=1$ and $c_{1}=c_{4}=0$ leads to the compatibility of the Hamiltonian operators (56).

Remark 6. The Hamiltonian pair $J$ and $K$ in each example in section 3 is a special case of the generalized form $J=c_{1} J_{1}+c_{2} J_{2}+c_{3} J_{3}$ and $K=c_{4} K_{1}+c_{5} K_{2}$, where $c_{1} c_{5}=c_{3} c_{4}$. The compatibility of such a Hamiltonian pair is guaranteed by proposition 1 .

\section{Conclusions and discussions}

In the paper, from the spectral problems (8) and (9), we propose a generalized two-component model (7) which allows for an arbitrary function $H$ to be involved in. We may generate many integrable peakon systems with different choices of $H$ in our model. So, our model provides a large class of peakon systems and covers almost all existing integrable peakon equations associated with $2 \times 2$ spectral problems. Because of the presence of an arbitrary function in the 
generalized system, we do not expect all those equations possess the bi-Hamiltonian structures in general. Nevertheless, we show that for some special choices of the function $H$ in (17) we may find the bi-Hamiltonian structures. Moreover, from the generalized model we obtain very interesting solutions, such as new type of $N$-peakon solution which is not in the traveling wave type.

Different from the usual integrable soliton equations, the peakon equation involved in an arbitrary function seems to be unusual. We believe that this system deserves a further investigation. The following two problems seem to be interesting:

- Is there a gauge transformation that can remove the arbitrary function $H$ ?

- Can the inverse scattering transforms be applied to solve our system in general?

Very recently, we know that Li, Liu and Popowicz [40] proposed a four-component peakon equation with an arbitrary function involved in, where they cited a preprint version [41] of the present paper. We believe that both our generalized peakon system and Li-Liu-Popowicz's system deserve a further investigation.

\section{ACKNOWLEDGMENTS}

The authors would like to express their sincerest thanks to the anonymous referee for the helpful suggestions and invaluable comments, which have helped us to improve this paper. The authors Xia and Zhou were supported by the National Natural Science Foundation of China (Grant Nos. 11301229 and 11271168), the Natural Science Foundation of the Jiangsu Province (Grant No. BK20130224) and the Natural Science Foundation of the Jiangsu Higher Education Institutions of China (Grant No. 13KJB110009). The author Qiao was partially supported by the National Natural Science Foundation of China (No. 11171295, No. 61301187, and No. 61328103) and also thanks the U.S. Department of Education GAANN project (P200A120256) to support UTPA mathematics graduate program.

\section{References}

[1] R. Camassa and D. D. Holm, An integrable shallow water equation with peaked solitons, Phys. Rev. Lett. 71 1661-1664 (1993).

[2] B. Fuchssteiner and A. S. Fokas, Symplectic structures, their Bäcklund transformation and hereditary symmetries, Physica D 4 47-66 (1981).

[3] P. J. Olver and P. Rosenau, Tri-Hamiltonian duality between solitons and solitary-wave solutions having compact support, Phys. Rev. E 53 1900-1906 (1996). 
[4] R. Beals, D. Sattinger and J. Szmigielski, Multipeakons and a theorem of Stieltjes, Inverse Problems 15 L1-L4 (1999).

[5] F. Gesztesy and H. Holden, Algebro-geometric solutions of the Camassa-Holm hierarchy, Rev. Mat. Iberoamericana 19 73-142 (2003).

[6] Z. J. Qiao, The Camassa-Holm hierarchy, N-dimensional integrable systems, and algebro-geometric solution on a symplectic submanifold, Commun. Math. Phys. 239 309-341 (2003).

[7] A. Constantin, On the scattering problem for the Camassa-Holm equation, Proc. R. Soc. Lond. Ser. A 457 953-970 (2001).

[8] A. Constantin and W. A. Strauss, Stability of peakons, Comm. Pure Appl. Math. 53 603-610 (2000).

[9] A. Constantin and D. Lannes, The hydrodynamical relevance of the Camassa-Holm and DegasperisProcesi equations, Arch. Ration. Mech. Anal. 192 165-186 (2009).

[10] R. Beals, D. Sattinger and J. Szmigielski, Multipeakons and the classical moment problem, Adv. Math. 154 229-257 (2000).

[11] M. S. Alber, R. Camassa, Y. N. Fedorov, D. D. Holm and J. E. Marsden, The complex geometry of weak piecewise smooth solutions of integrable nonlinear PDEs of shallow water and Dym type, Commun. Math. Phys. 221 197-227 (2001).

[12] R. S. Johnson, On solutions of the Camassa-Holm equation, Proc. R. Soc. Lond. A 459 1687-1708 (2003).

[13] A. Degasperis and M. Procesi, Asymptotic integrability, in Symmetry and perturbation theory (eds A Degasperis, G Gaeta), River Edge, NJ: World Scientific Publishing, pp. 23-37, 1999.

[14] A. Degasperis, D. D. Holm and A. N. W. Hone, A new integrable equation with peakon solitons, Theor. Math. Phys. 133 1463-1474 (2002).

[15] H. Lundmark and J. Szmigielski, Multi-peakon solutions of the Degasperis-Procesi equation, Inverse Problems 19 1241-1245 (2003).

[16] J. Szmigielski, L. J. Zhou, Colliding peakons and the formation of shocks in the Degasperis-Procesi equation, Proc. R. Soc. A 46920130379 (2013).

[17] A. S. Fokas, On a class of physically important integrable equations, Physica D 87 145-150 (1995).

[18] B. Fuchssteiner, Some tricks from the symmetry-toolbox for nonlinear equations: generalizations of the Camassa-Holm equation, Physica D 95 229-243 (1996).

[19] Z. J. Qiao, A new integrable equation with cuspons and W/M-shape-peaks solitons, J. Math. Phys. 47112701 (2006).

[20] G. L. Gui, Y. Liu, P. J. Olver and C. Z. Qu, Wave-breaking and peakons for a modified CamassaHolm equation, Commun. Math. Phys. 319 731-759 (2013).

[21] V. Novikov, Generalizations of the Camassa-Holm equation, J. Phys. A: Math. Theor. 42342002 (2009). 
[22] A. N. W. Hone and J. P. Wang, Integrable peakon equations with cubic nonlinearity, J. Phys. A: Math. Theor. 41372002 (2008).

[23] Z. J. Qiao, B. Q. Xia and J. B. Li, Integrable system with peakon, weak kink, and kink-peakon interactional solutions, arXiv:1205.2028v2.

[24] M. Chen, S. Q. Liu and Y. Zhang, A two-component generalization of the Camassa-Holm equation and its solutions, Lett. Math. Phys. 75 1-15 (2006).

[25] G. Falqui, On a Camassa-Holm type equation with two dependent variables, J. Phys. A 39 327-342 (2006).

[26] D. D. Holm and R. I. Ivanov, Multi-component generalizations of the CH equation: geometrical aspects, peakons and numerical examples, J. Phys A: Math. Theor. 43492001 (2010).

[27] J. F. Song, C. Z. Qu and Z. J. Qiao, A new integrable two-component system with cubic nonlinearity, J. Math. Phys. 52013503 (2011).

[28] B. Q. Xia and Z. J. Qiao, A new two-component integrable system with peakon solutions, Proc. R. Soc. A 47120140750 (2015).

[29] X. G. Geng and B. Xue, An extension of integrable peakon equations with cubic nonlinearity, Nonlinearity 22 1847-1856 (2009).

[30] A. Degasperis, D. D. Holm, A. N. W. Hone, Integrable and non-integrable equations with peakons, in: Proceedings of Nonlinear Physics-Theory and Experiment II, World Scientific, pp. 37-43, 2002.

[31] D. D. Holm and M. Staley, Wave structure and nonlinear balance in a family of $1+1$ evolutionary PDE's, SIAM J. Appl. Dyn. Syst. 2 323-380 (2003).

[32] A. V. Mikhailov and V. S. Novikov, Perturbative symmetry approach, J. Phys. A 35 4775-4790 (2002).

[33] A. N. W. Hone and J. P. Wang, Prolongation algebras and Hamiltonian operators for peakon equations, Inverse Problems 19 129-145 (2003).

[34] A. N. W. Hone, Painlevé tests, singularity structure and integrability, in: A.V. Mikhailov (Ed.), Integrability, in: Lect. Notes Phys. 767 Springer, Berlin, Heidelberg, pp. 245-277, 2009.

[35] A. N. W. Hone and S. Lafortune, Stability of stationary solutions for nonintegrable peakon equations, Physica D 269 28-36 (2014).

[36] Z. Popowicz, A two-component generalization of the Degasperis-Procesi equation, J. Phys. A: Math. Gen. 39 13717-13726 (2006).

[37] A. N. W. Hone and M. V. Irle, On the non-integrability of the Popowicz peakon system, Discrete Cont. Dyn. S. Supplement pp. 359-366 (2009); e-Print, arXiv: 0808.2617.

[38] H. Lundmark and J. Szmigielski, An inverse spectral problem related to the Geng-Xue twocomponent peakon equation, arXiv: $1304.0854 \mathrm{v} 1$. 
[39] K. Tian and Q. P. Liu, Tri-Hamiltonian duality between the Wadati-Konno-Ichikawa hierarchy and the Song-Qu-Qiao hierarchy, J. Math. Phys. 54043513 (2013).

[40] N. Li, Q. P. Liu and Z. Popowicz, A four-component Camassa-Holm type hierarchy, J. Geom. Phys. 85 29-39 (2014).

[41] B. Q. Xia, Z. J. Qiao and R. G. Zhou, A synthetical integrable two-component model with peakon solutions, 2013, arXiv: 1301.3216. 